\title{
Acute erythroblastic leukemia (AEL): a rare subset of de novo AML with a complex rearrangement involving ETV6 locus and loss of RBI locus
}

\section{Editorial}

Acute Erythroid leukemia (AEL) is a rare subset of acute myeloid leukemia (AML). AEL has been reported mostly in adults and comprises less than $5 \%$ of AML (WHO, 2008). In addition, AEL has been further divided into erythroid leukemia (erythroid/myeloid) and pure erythroid leukemia. ${ }^{1,2}$ Pure erythroid leukemia consists of neoplastic proliferation of immature cells that are committed to erythroid lineage has been reported (WHO, 2008). Pure erythroid leukemia can occur at any stage. The genetic abnormalities that have been reported in AEL are similar to those identified in Acute Myeloid leukemia (AML) with myelodysplastic (MDS) changes and consists of complex karyotype with structural abnormalities including -5/ $\operatorname{del}(5 q),-7 / \operatorname{del}(7 q),+8$ and/or RUNX1 mutations. However, JAK2, FLT3, TP53 and RAS mutations have been reported to be of low incidence in AEL. ${ }^{3}$ The prognosis has been described unfavorable with complex karyotype.

Cryptic and recurrent rearrangement of $\mathrm{t}(7 ; 12)$ involving ETV6 locus has been identified in children of age two years or younger with various types of leukemia. Heterogeneity of the breakpoints ranging from $7 \mathrm{q} 32$ (very few) or q36 (frequently) and 12p13 have been reported in myeloid disorders. ${ }^{4-9}$ We report here a case of Pediatric Acute Erythroblastic Leukemia with a variant $\mathrm{t}(7 ; 12)$ translocation involving three chromosomes 5, 7 and 12 with loss of the RB1 locus at 13q14 region of chromosome 13. Sequential metaphase FISH and cytogenetic were performed to identify the partner chromosomes in the ETV6 locus rearrangement.

A two- year old male with a fatigue, bruising, petechiae and fever was presented for evaluation of normocytic anemia and thrombocytopenia in February, 2014. His initial bone marrow (BM) biopsy was hyper cellular, packed with a predominance of erythroids and a large percentage of immature forms. Flow cytometry was positive for $\mathrm{CD} 33, \mathrm{CD} 7, \mathrm{CD} 36$ and $\mathrm{CD} 71$, consistent with erythroid differentiation of immature cells. The diagnosis was FLT3-, NMP1and KIT17 D816V negative AML. Cytogenetics revealed seventeen of the twenty cells analyzed revealed an abnormal karyotype and three cells were normal. Karyotype was highly complex with 46,XY,der(5) $\operatorname{del}(5)(\mathrm{p} 15.1 \mathrm{p} 15.1) \mathrm{t}(5 ; 12 ; 7), \operatorname{der}(7) \mathrm{t}(5 ; 12 ; 7), \operatorname{der}(12) \operatorname{del}(12)(\mathrm{p} 13 \mathrm{p} 13)$ $\mathrm{t}(5 ; 12 ; 7), \quad \operatorname{del}(13)(\mathrm{q} 12 \mathrm{q} 14)[17] / 46, \mathrm{XY}[3]$ with a simultaneous deletion of $5 \mathrm{p} 15.1$ and $12 \mathrm{p} 13$ regions of derivative chromosome 5 and derivative respectively, along with an interstitial deletion of chromosome 13 from q12q14 region involving the RB1 locus (Figure 1-3). The patient entered remission with induction chemotherapy, received consolidation chemotherapy, and at most recent follow up of about 18 months after the initial diagnosis bone marrow studies were negative for leukemia by morphology and cytogenetic.

The variant translocation described here has not been previously reported. The putative disrupted gene in this translocation at $7 \mathrm{q} 32$ is unknown. This breakpoint at $7 \mathrm{q} 32$ appears to have a better prognosis than translocations involving the $7 \mathrm{q} 36$ locus, as most patients with the $7 \mathrm{q} 36$ breakpoint have had a poor outcome. This is also the first

\author{
Volume 2 Issue 2 - 2016
}

\section{Yenamandra A, Hollis A, Zalepa D, Head D Department of Pathology, Immunology and Microbiology, Vanderbilt University Medical Center, Nashville, USA}

\begin{abstract}
Correspondence: Ashwini Yenamandra, Medical Director of Clinical Cytogenetics, Assistant Professor, Department of Pathology, Immunology and Microbiology, Vanderbilt University Medical Center 719,Thompson Lane, Nashville, TN 37204, USA, Email ashwini.yenamandra@vanderbilt.edu
\end{abstract}

Received: January 26, 2016 | Published: February 25, 2016

case report with a deletion of RB1 locus in AEL. A thorough literature search including Mitelman database of Chromosome Aberrations and Gene Fusions in Cancer ${ }^{4-10}$ did not reveal report of cases with similar complex rearrangement of three chromosomes and deleted chromosome13q.

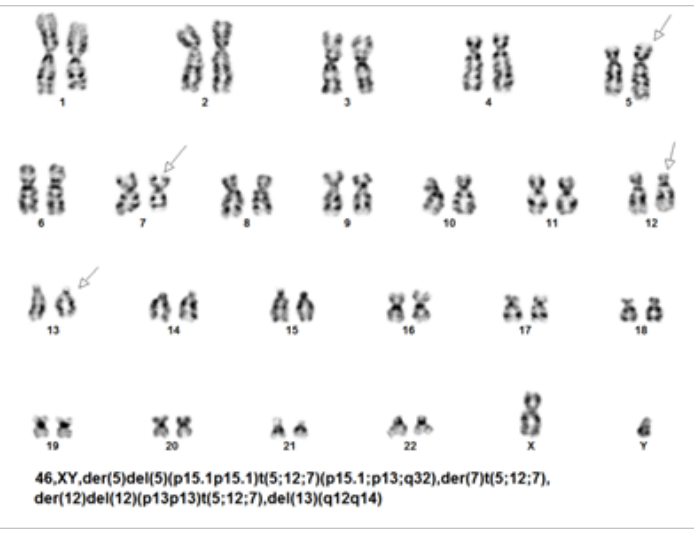

Figure I Abnormal Karyotype.

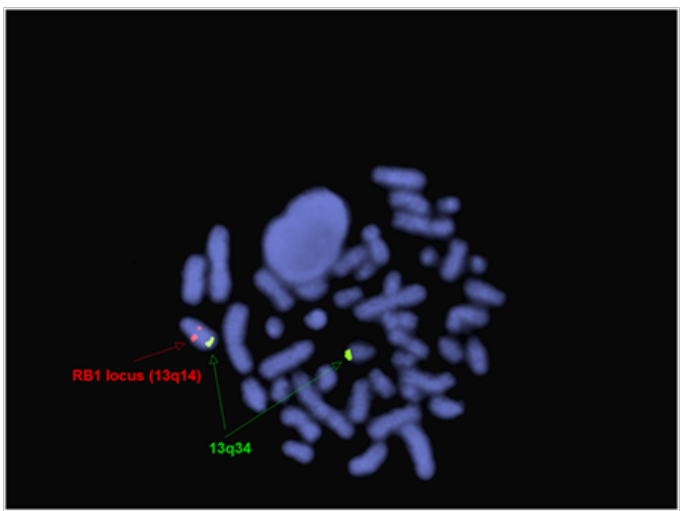

Figure 2 Deletion of RBI locus. 


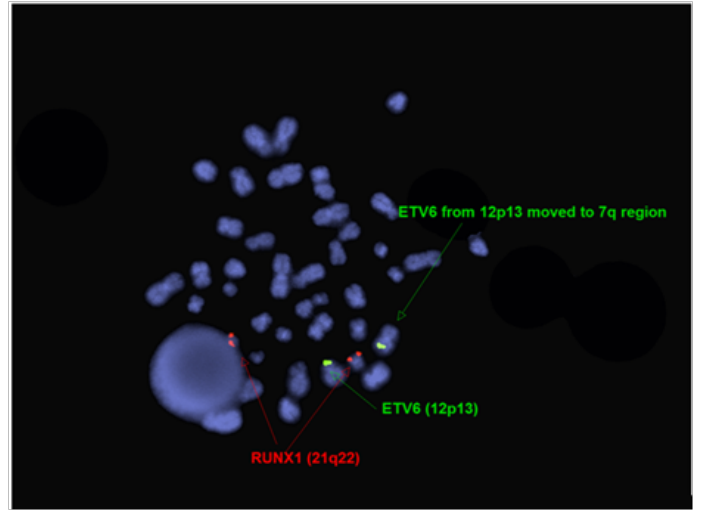

Figure 3 ETV6 from $12 p / 3$ region moved to $7 q$.

\section{Conclusion}

In summary, we present a case with a novel rearrangement involving the important hematopoietic regulatory gene ETV6 in a case of pediatric primary AML. AEL is a rare subset of AML and a complex and variant $\mathrm{t}(7 ; 12)$ with ETV6 rearrangement involving 8 three chromosomes and loss of RB1 is a rare cytogenetic finding; this combination in pediatric primary de novo AEL and importance of the regulatory role of ETV6 gene in hematopoiesis is unique in the 9 literature.

\section{Acknowledgements}

None.

\section{Conflict of interest}

The author declares no conflict of interest.

\section{References}

1. Mihova D, Zhang L. Acute erythroid leukemia : A Review. North American Journal of Medicine and Science. 2012;5(2):110-118.

2. Santos FPS, Bueso-ramos CE. Acute erythroleukemia: diagnosis and management. Expert Rev Hematol. 2010;3(6):705-718.

3. Sara T, Barbara C, Benjamin T. An uncommon variant of acute myeloid leukemia: acute erythroid Leukemia. Lab Medicine. 2011;42(11):644-648.

4. Farhat AB, Iftikhar H, Muhammad ZA. Adult B lymphoblastic leukaemia/ lymphoma with hypodiploidy (-9) and a novel chromosomal translocation $\mathrm{t}(7 ; 12)(\mathrm{q} 22 ; \mathrm{p} 13)$ presenting with severe eosinophilia-case report and review of literature. J Hematol Oncol. 2009;2:26.

5. Park J, Kim M, Lim J, et al. Three-way complex translocations in infant acute myeloid leukemia with $\mathrm{t}(7 ; 12)(\mathrm{q} 36 ; \mathrm{p} 13)$ : the incidence and correlation of a HLXB9 overexpression. Cancer Genet Cytogenet. 2009;191(2):102-105.

6. Slater RM, von Drunen E, Kroes WG, et al. t(7;12)(q36;p13) and t(7;12) (q32;p13) translocations involving ETV6 in children 18 months of age or younger with myeloid disorders. Leukemia. 2001;15(6):915-920.

7. Tosi S. t(7;12)(q36;p13). Atlas Genet Cytogenet Oncol Haematol. 2001;5:195-196

Tosi S, Hughes J, Scherer SW, et al. Heterogeneity of the $7 \mathrm{q} 36$ breakpoints in the $\mathrm{t}(7 ; 12)$ involving ETV6 in infant leukemia. Genes Chromosomes Cancer. 2003;38:191-200.

Von Bergh ARM, Beverloo HB. t(7;12)(q36;p13) Atlas genet cytogenet oncol haematol. 2004;8:85-87.

10. http://cgap.nci.nih.gov/Chromosomes/AbnCytSearchForm 